\section{Sclerofoam assisted laser therapy for saphenous refluxes: an innovative tumescence-free technique}

\author{
Francesco Zini, ${ }^{1}$ Lorenzo Tessari, ${ }^{2}$ \\ Renato Torre ${ }^{3}$ \\ 'Surgical Department, Casa di Cura Città \\ di Parma, Parma; ${ }^{2}$ Glauco Bassi \\ Foundation, Trieste; ${ }^{3}$ Casa di Cura \\ Privata, Piacenza, Italy
}

\section{Abstract}

Endovenous laser (EL) and radiofrequency devices have continuously increased their appealing in the last decade. Even if miniinvasive, such procedure still requires multiple high volume injections of tumescent anesthesia: a medical act that is not totally complications-free. Aim of the present investigation is to evaluate the feasibility of a hybrid technique (so called sclerofoam assisted laser therapy, SFALT) combining foam sclerotherapy (FS) and EL in a tumescence free approach. Fourty primary chronic venous disease patients (8 males, 32 females, C2-4EpAsPr) presenting a sapheno-femoral reflux both at the Valsalva and compression/relaxation maneuver underwent a SFALT procedure. Diameters were measured at mid-thigh in supine. It consists in a EL fiber introduction into the great saphenous vein (GSV), shrinking it for a single $\mathrm{cm}$ at $200 \mathrm{~J} / \mathrm{cm}$. After a shrunk plug is created, keeping the fiber stuck in it, $5 \mathrm{cc}$ of foam sclerotherapy [Tessari method, $1 \%$ polidocanol (POL) or $1 \%$ sodium tetradecyl sulfate (STS)] are injected through the same 6 Fr EL introducer. The consequent spasm allows a following EL mediated shrinkage by means of a significantly reduced fluence. Clinical and sonographic follow up were performed at one and three weeks. At 3 weeks follow up all the 40 cases presented a shrunk GSV, without recanalization signs. Neither major nor minor complications were reported. At the mid-thigh the standing GSV caliber decreased from a preoperative mean value of $0.6 \pm 0.2 \mathrm{~cm}$ to a post FS injection $0.3 \pm 0.1 \mathrm{~cm}$ value $(\mathrm{P}<0.05)$, showing no statistical difference among STS and POL. SFALT approach is feasible, safe and with potentially interesting outcomes. More investigations are needed in order to define the proper fluence parameters and the chance of eliminating the even mild sedation. This technique offers the chance of a possible tumescence free GSV treatment, even in case of major calibers vessels.

\section{Introduction}

Endovenous laser (EL) and radiofrequency (RF) devices have continuously increased their appealing in the last decade, so much to be recommended with a $1 \mathrm{~B}$ grade in the most recent guidelines. ${ }^{1}$ Thanks to its ease of use, fast procedural time, safety and effectiveness profiles, endovenous procedures have been progressively substituting in most counties the traditional surgical stripping. ${ }^{2}$

Even if mini-invasive, such a procedure still requires multiple high volume injections of tumescent anesthesia (TA). TA represents a relatively safe act. Nevertheless it is not only responsible for quite easily observed painful and not aesthetic hematomas along the saphenous area, but is also not free from potential complications. ${ }^{3-5}$ At the same time, foam sclerotherapy (FS) has proved to represent an extremely valid alternative therapeutic tool, since the diffusion of a powerful, cost-effective and easily reproducible extemporary production method. ${ }^{6,7}$

A brand new holmium laser device has been developed and combined with FS in order to avoid TA and being effective even in a large diameter great saphenous vein (GSV), demonstrating preliminary promising outcomes. ${ }^{7}$

\section{Objectives}

Aim of the present investigation is to explore the feasibility of a FS assisted-traditional EL use in absence of TA exploiting the spasm effect that is caused by the sclerosing drug. Secondary endpoint is the exploration of new EL energy deliver settings, eventually significantly reduced thanks to the synergic FS action, thus leading the procedure to a simplified office-based scenario.

\section{Materials and Methods}

Forty chronic venous disease patients with a symptomatic GSV incompetence (8 males; 32 females; C2-4EpAsPr C2(17), C3(20), C4(3); mean age was $51 \pm 8$ years old; body mass index $22 \pm 4$ ) underwent a standing-up pre-operative ultrasound (US) scanning demonstrating the homogeneity in the reflux pattern (saphenofemoral junction incompetence placing the sample volume at the femoral side of terminal valve detected by both positive Valsalva and compression/relaxation maneuver, single or multiple incompetent saphenous tributaries along the lower limb). A normally developed incompetent saphenous trunk (anatomically found always in between the fascia splitting, according to the so called saphenous eye $)^{8}$ was present all the way from the sapheno-femoral junction to below the knee.
Correspondence: Francesco Zini, Surgical Department, Casa di Cura Città di Parma, p.zza A. Maestri 5, 43123 Parma, Italy

Tel.: +39.0521.284472.

E-mail: fzflebo@tin.it

Key words: Tumescense; laser; foam.

Received for publication: 7 March 2015.

Revision received: 17 April 2015.

Accepted for publication: 17 April 2015.

This work is licensed under a Creative Commons Attribution 3.0 License (by-nc 3.0).

(O) Copyright F. Zini et al., 2015

Licensee PAGEPress, Italy

Veins and Lymphatics 2015; 4:5141

doi:10.4081/vl.2015.5141

No pelvic varicose veins were reported.

Diameters were measured at mid-thigh in supine to evaluate the foam injection-induced spasm. A second scanning was repeated immediately before the procedure, GSV diameters were recorded below the superficial epigastric vein and at the middle thigh, in supine. After a mild intra-venous sedation with dosages related to the short procedural time and limited pain trigger (midazolam $3 \pm 1 \mathrm{cc}$ ), the procedure included a percutaneous GSV access without local anesthesia at the distal third of the thigh or below the knee, depending on the incompetent tributary localization, a 0.0035 -in guide-wire insertion, placement of a 6 Fr introducer, insertion of $1470 \mathrm{~nm}$ diode laser radial fiber $600 \mu \mathrm{m}$ up to $1 \mathrm{~cm}$ below the superficial epigastric vein confluence. Particular care was used in detecting the anterior accessory saphenous vein confluence with the GSV and its eventual incompetence that potentially could be treated in the same procedural session. All patients were accurately informed about the procedure, according to international rules.

The EL was then activated $(6-8 \mathrm{~W}, 30-50$ $\mathrm{J} / \mathrm{cm}$ ), shrinking the GSV by a $200 \mathrm{~J}$ fluence for the first $\mathrm{cm}$ in order to create a shrunk plug, completely obliterating the vessel in that limited segment.

Keeping the fiber tip tucked into the shrunk plug, 5 cc of FS 1:4 (drug/air ratio according to the Tessari method, using two $5 \mathrm{~mL}$ siliconefree syringes), were then injected directly by the 3 -way introducer using $1 \%$ polidocanol (POL) (30 cases of whom 5 males, 25 females) or $1 \%$ sodium tetradecyl sulfate (STS) (10 cases of whom 3 males and 7 females) (Figure 1). A finger compression below the introducer insertion point guaranteed the FS upward flow.

The injected foam was clearly detected echographically getting up to the shrunk plug and then filling up the minor GSV tributaries (Figure 2). 
A sudden venous spasm is obtained, optimizing the subsequent EL shrinkage that was performed all the way down to the knee GSV region with a significant reduction of the usual fluence. Varicose veins that were not previously reached by the injected foam can than be treated both by phebectomies or FS itself, as occurred in 3 patients of our study population. An above-knee $20-30 \mathrm{mmHg}$ elastic stocking compression was prescribed to all the patients for one week $24 \mathrm{~h}$ a day, then just during the daytime for the following two weeks.

All the patients underwent an immediately post-operative US and clinical follow-up, that was repeated by the same assessor at 7 and 21 days.

\section{Results}

In supine at the mid-thigh the GSV caliber decreased from a pre-operative mean value of $0.6 \pm 0.2 \mathrm{~cm}$ to a post $\mathrm{FS}$ injection $0.3 \pm 0.1 \mathrm{~cm}$ value $(\mathrm{P}<0.05)$, showing no statistical difference among STS and POL $(\mathrm{P}=0.7)$.

At the two follow-up visits (7 and 21 days after the procedure) all the patients presented a GSV shrinkage with reflux suppression. Neither major nor minor complications were reported. In particular neither ecchymosis nor hematoma were detected at the thigh.

No significant pain was reported by the patients, clearly demonstrating the feasibility of the herein presented tumescence-free technique.

\section{Discussion and Conclusions}

EL and RF have progressively gathered a major role in therapeutic international indications for varicose veins treatment.

Nevertheless, the same guidelines point out the need of a smaller than $15 \mathrm{~mm}$ caliber GSV for a RF approach, while stating that, even if there are no absolute contraindicated GSV calibers for EL, ${ }^{9}$ an association among larger than $8 \mathrm{~mm}$ GSV and femoral thrombus extension was reported. ${ }^{10,11}$

EL is scarless, aesthetically satisfying and considered as a mini-invasive effective therapeutic approach. Nevertheless, up to now, EL is strictly related to TA.

This last one has four main aims: cooling and protecting the perivascular tissue from heating and burns, pain control, guaranteeing at least $1 \mathrm{~cm}$ of depth from the skin surface, reducing the GSV caliber in order to remove blood and improving surface contact.

On the other side, eve if minimally, TA is an invasive act that can be related to unpleasant consequences such as perivenous ecchymosis and hematoma, up to $52 \%$ of patients, all along the treated GSV tract. ${ }^{12,13}$

This fact is responsible for a temporarily aesthetic impact, involving the patient dissatisfaction.

Rare but extremely severe TA complications have also been described in the literature..$^{2-4}$

At the same time the same TA injection technique can present some difficulties leading to complications. In the same way, if the injecting needle remains too far away from the vein the perivenous tissue will remain attached to the vein, so suffering of heat damage. ${ }^{5}$ Nowadays, technological innovations are developing in order to eliminate the TA need. In particular, a holmium laser assisted foam sclerotherapy (LAFOS) has been introduced with definitely promising outcomes. ${ }^{7}$ Thanks to the low temperature generated by LAFOS a GSV caliber reduction is made possible even at the lowest energy delivery, so avoiding the need of any kind of TA. A following foam sclerotherapy injection completes the venous shrinkage.

The herein reported absence of peri-operative and early post-operative complications points out how our strategic proposal produces preliminary evidences regarding the feasibility of a new EL use without the need of both any TA and of any new laser device.

The FS introduction in a previously partially shrunk GSV induces a venous spasm around the fiber, creating an endothelial damage, so allowing a lower EL energy delivery.

Moreover, the creation of a shrinkage plug on the cranial saphenous side, limit the diffusion of FS derived cathabolites (like endothelin and histamin), maximizing the same drug local effect, while minimizing the systemic possible even if extremely rare side effects. ${ }^{14}$

The synergic EL $1470 \mathrm{~nm}$ and FS effect, thanks to the more available water cromophore (water is specific target of $1470 \mathrm{~nm}$ laser frequency), may be that paves the way for a brand new definition of the energy delivery parameters in endovenous techniques, with hypothetical consequent smaller side effects and higher patient satisfaction, providing a TA-free strategy to be performed in the future in an officesetting. Further investigations to this feasibility study are ongoing regarding the EL energy delivery settings and the spasm effect induced by different concentrations of POL $v s$ STS in a wider and thus more significant patient population, in absence we hope also of any kind of sedation. Further investigations are needed for the proposed strategy application both in larger GSV calibers and with the use also of RF devices.

This study is a feasibility study. Number of patients and, in particular, follow-up time are clearly insufficient to draw conclusions. A more complex and accurate study is in progress.

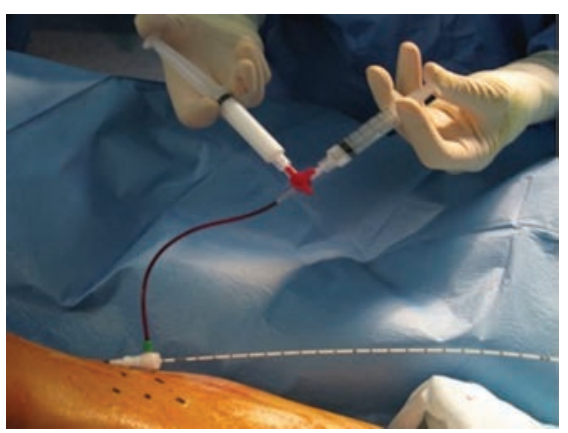

Figure 1. Foam sclerotherapy preparation and injection through the same laser fiber introducer. Laser fiber remains endovenous, blocked by the initially performed shrunk plug.

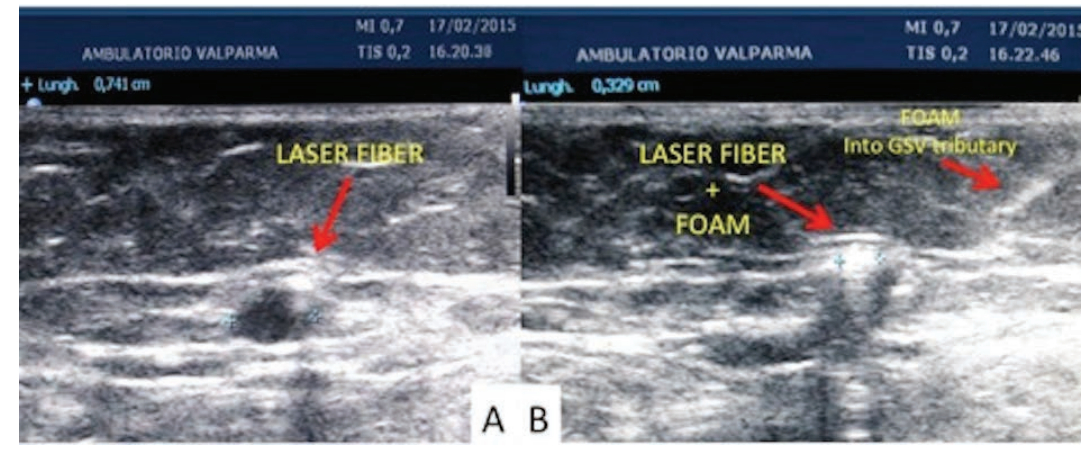

Figure 2. A) Laser fiber into the great saphenous vein (GSV) after a shrunk plug was created just below the superficial epigastric vein confluence, sparing the remaining GSV trunk. B) Foam injection into the GSV with the laser fiber still inside the vein. A consequent significant caliber decrease is detected after the injection. Foam easily reaches the GSV tributaries along the thigh thanks to the block caused by the shrunk plug. 


\section{References}

1. Gloviczki P, Comerota AJ, Dalsing MC, et al. The care of patients with varicose veins and associated chronic venous diseases: clinical practice guidelines of the Society for Vascular Surgery and the American Venous Forum. J Vasc Surg 2011;53:2S-48S.

2. Khilnani NM, Winokur RS. Varicose vein treatment with endovenous laser therapy. Medscape; Apr 10, 2014. Available from: http://emedicine.medscape.com/article/18 15850-overview

3. Holdstock JM, Marsh P, Whiteley MS, Price BA. It is possible to cause damage to a laser fibre during delivery of tumescent anaesthesia for endovenous laser ablation (EVLA). Eur J Vasc Endovasc Surg 2008;36: 473-6.

4. Hubmer MG, Koch H, Haas FM, et al. Necrotizing fasciitis after ambulatory phlebectomy performed with use of tumescent anesthesia J Vasc Surg 2004;39:263-5.
5. Memetoglu ME, Kurtcan S, Kalkan A, Özel D. Combination technique of tumescent anesthesia during endovenous laser therapy of saphenous vein insufficiency. Interact CardioVasc Thorac Surg 2010;11: 774-8.

6. Cavezzi A, Tessari L. Foam sclerotherapy techniques: different gases and methods of preparation, catheter versus direct injection. Phlebology 2009;24:247-51.

7. Coleridge Smith P. Sclerotherapy and foam sclerotherapy for varicose veins. Phlebology 2009;24:260-9.

8. Caggiati A. Fascial relationship of the long saphenous vein. Circulation 1999;100: 2547-9.

9. Frullini A, Fortuna B. Laser assisted foam sclerotherapy (LAFOS): a new approach to the treatment of incompetent saphenous veins. Phlébologie 2013;66:51-4.

10. Florescu C, Curry G, Buckenham T. Role of endovenous laser therapy in large and very large diameter great saphenous veins. ANZ J Surg 2014 [Epub ahead of print].
11. Lawrence PF, Chandra A, Wu M, et al. Classification of proximal endovenous closure levels and treatment algorithm. $\mathrm{J}$ Vasc Surg 2010;52:388-93.

12. Chaar CI, Hirsch SA, Cwenar MT, et al Expanding the role of endovenous laser therapy: results in large diameter saphenous, small saphenous, and anterior accessory veins. Ann Vasc Surg 2011; 25:656-61.

13. Vaz C, Matos A, Sameiro M, et al. Iatrogenic complications following laser ablation of varicose veins. In: Yamanaochi D, ed. Vascular surgery. Rijeka: InTech; 2012. Available from: http://www.intechopen.com/books/vascular-surgery/iatrogenic-complications-following-laser-ablation-of-varicose-veins-

14. Frullini A, Barsotti MC, Santoni T, et al. Significant endothelin release in patients treated with foam sclerotherapy. Dermatol Surg 2012;38:741-7. 